development is the attempt towards side effect reduction. Successful examples are comedication with folic acid during methotrexate treatment and of biphosphonate during corticosteroid treatment.

- Target oriented therapies: whereas anti-T cell therapies so far have not been consistently successful, TNF blocking agents have revolutionised treatment of rheumatoid arthritis. These agents work fast and give a good response in over half of the patients. In addition it has been shown that these therapies have the potential of stopping (radiographic) joint damage. From a practical point of view there are at least a few problems: these therapies are rather expensive and long term follow-up data are not yet available. Especially the latter is important, since TNF plays an important role in inflammation and immunity and thefore chronic suppression may also have negative consequences. Furthermore, it is advised that during TNF blocking therapies, doctor and patiënt should be alert on the presence or development of infectious diseases.

In view of the above considerations, current use of TNF blocking therapies should be restricted to patiënts with active disease, despite adequate DMARD therapy, including methotrexate. Possible future strategies will be discussed.

\section{SP0112 EXPERIENCES FROM A NATIONAL SURVEILLANCE PROGRAM FOR EFFECTS, SIDE-EFFECTS AND COSTS OF TNF-BLOCKING AGENTS IN SWEDEN}

N Feltelius. Division of Clinical Trials, Medical Products Agency, Uppsala, Sweden

10.1136/annrheumdis-2001.50

The introduction of TNF-blockers in rheumatology has focused on the need for new systems for follow-up of new drugs. Many drugs, like the TNF-blockers have new mechanisms of actions and thus unknown side effects. The national influence on the approval of new drugs has decreased with the common European regulatory system. However the economic consequences of these often expensive treatments are to be handled on the national level. Thus for medical as well as economical reasons national efforts to collect information about recently marketed drugs are important.

In a joint project the Swedish Medical Products Agency (MPA) and The Swedish Society for Rheumatology introduced a follow-up system for the new TNF-blockers when used in rheumatoid arthritis. The system was implemented already before marketing. The aim is to elaborate a system for collecting and reporting pharmacotherapy data from all rheumatology units in Sweden to assess safety and efficacy. This is to optimise treatment but also to form the basis for future surveillance programmes for other new drugs.

The study started in early 1999 with a planned 2-year duration. Assessments of patients are scheduled at 0,3, 6, 12, 18 and 24 months. Patients included are those with active RA who has failed previous DMARD treatment. All patients are treated on a named-patient-basis and are identified by a unique personal number. This system makes possible future cross-linking with other health data bases. Disease activity is measured according to EULAR core set. The concomitant use of DMARDs and other medications is recorded in detail.

Treatment reports have till now been collected from 1280 patients. The overall clinical outcome of etanercept as well as of infliximab treatment is much in line with what has been reported from randomised controlled studies (RCTs). Only a small number of patients has been followed for over 12 months but efficacy seems sustained also after this time point.

Reporting of adverse events (AEs) is higher in this surveillance study than what can be expected from the regular spontaneous reporting system. In total 425 AEs have been reported in the cohort. Most frequent are injection site reactions and other dermatological manifestations. Among serious adverse events infections were predominant, with cases of septic arthritis and septicaemia reported. The cost for TNF-blocker treatment in Sweden during the year 2000 is 170 million SK. The spending calculated per inhabitant indicate substancial regional differences in prescription. These figures includes treatment of Crohn's disease patients.

The main conclusions from this study are: 1 . Results of TNFblocker treatment from RCTs seem valid also in an everyday clinical setting. 2. Reporting of adverse events can be substantially improved compared to the existing systems. 3 . The logistics of reporting was the main problem in establishing the data collecting system. 4. The involvement of the MPA and the Swedish Society for Rheumatology was necessary to implement the system. 5. For the future the establishing of firm and independent systems that can further develop these kind of drug surveillance is highly desirable. In this struggle the participation of all key players regulatory agencies, professional societies, academic institutions and drug companies is necessary.

\section{Metabolic disorders and the musculoskeletal system - Saturday 16 June, 12.00-13.30/ Forum Hall}

\section{SP0115 CRYSTALS AND JOINTS}

M Doherty. Academic Rheumatology, City Hospital, Nottingham, UK

10.1136/annrheumdis-2001.51

Primary metabolic disease may predispose to the deposition of a variety of crystals in and around joints, most notably calcium pyrophosphate (CPPD) and apatite. Crystal associated musculoskeletal symptoms may be the presentation of such disease. Renal failure additionally predisposes to urate and oxalate crystal deposition. In most disease states the principal mechanisms relate to elevated ionic products.

There is good evidence that hyperparathyroidism, hypomagnesaemia, haemochromatosis and hypophosphatasia predispose to CPPD deposition; data are less convincing for hypothyroidism, Wilson's disease and other putative associations. Clinical features are acute "pseudogout" attacks in association with polyarticular chondrocalcinosis (CC). Haemochromatosis is exceptional in additionally causing structural joint change and chronic symptoms. Patients $<55$ years presenting with recurrent acute attacks and polyarticular CC should be screened for metabolic disease (minimum $=$ serum ferritin, magnesium, calcium, alkaline phosphatase). Treatment of the primary disease, however, probably little influences the outcome of CPPD and CC. All these diseases increase extracellular pyrophosphate levels; effects on CPPD crystal nucleation and dissolution, however, are also evident. Rare monogenic familial CC may shortly be explained as primary metabolic disorders of pyrophosphate metabolism involving several enzyme systems. 